\title{
On the Angular Density of Three Dimensional Scattering Resonances
}

\author{
Lung-Hui Chen \\ Department of Mathematics, National Chung Cheng University, 168 University Road, Min-Hsiung, Chia-Yi 621, Taiwan
}

Correspondence should be addressed to Lung-Hui Chen; mr.lunghuichen@gmail.com

Received 1 January 2014; Accepted 4 February 2014; Published 9 March 2014

Academic Editors: N. E. Bjerrum-Bohr, D. Dürr, A. L. Salas-Brito, and F. Sugino

Copyright (c) 2014 Lung-Hui Chen. This is an open access article distributed under the Creative Commons Attribution License, which permits unrestricted use, distribution, and reproduction in any medium, provided the original work is properly cited.

We apply Cartwright's theory in integral function theory to describe the angular distribution of scattering resonances in mathematical physics. A quantitative description on the counting function along rays in complex plane is obtained.

\section{Introduction}

In this paper, we study the distribution of the scattering resonances of a certain class of elliptic operators arousing from Schrödinger operator. We have

$$
H:=\Delta_{\mathbb{R}^{3}}+V: L^{2}\left(\mathbb{R}^{3}\right) \longrightarrow L^{2}\left(\mathbb{R}^{3}\right),
$$

where $V \in \mathscr{C}_{0}^{\infty}\left(\mathbb{R}^{3} ; \mathbb{R}\right)$. Let us denote the physical plane by

$$
\mathscr{P}:=\{\lambda \in \mathbb{C} \mid \Im \lambda>0\} .
$$

It is well-known from spectral analysis that the resolvent operator $\left(H-\lambda^{2}\right)^{-1}: L^{2}\left(\mathbb{R}^{3}\right) \rightarrow H^{2}\left(\mathbb{R}^{3}\right)$ is bounded in $\mathscr{P}$ except for some finite set $\left\{\mu_{1}, \ldots, \mu_{e}\right\}$ such that $\left\{\mu_{1}^{2}, \ldots, \mu_{e}^{2}\right\}$ are the pure point spectrum of $H$. The resolvent $\left(H-\lambda^{2}\right)^{-1}$ can be meromorphically extended from $\mathscr{P}$ to $\mathbb{C}$ as an operator:

$$
R(\lambda):=\left(H-\lambda^{2}\right)^{-1}: L_{\text {comp }}^{2}\left(\mathbb{R}^{3}\right) \longrightarrow H_{\text {loc }}^{2}\left(\mathbb{R}^{3}\right)
$$

with poles of finite rank. All such meromorphic poles in $\mathbb{C}$ are called resolvent resonances in mathematical physics literature. There are scattering theories in more generalized formalism. We refer to [1-3]. Let all of the meromorphic poles of $R(\lambda)$ be denoted as

$$
\mathscr{R}:=\left\{\mu_{1}, \ldots, \mu_{e}, \lambda_{1}, \lambda_{2}, \ldots\right\}
$$

repeated according to the multiplicity such that the only accumulation point is at infinity. The possible infinite set $\left\{\lambda_{1}, \lambda_{2}, \ldots\right\}$ is in the lower half complex plane.
The resolvent operator $R(\lambda)$ defines a scattering matrix

$$
S(\lambda): L^{2}\left(\mathbb{S}^{2}\right) \longrightarrow L^{2}\left(\mathbb{S}^{2}\right),
$$

which is of the form $I+A(\lambda)$, where $A(\lambda)$ is of trace class depending meromorphically on $\lambda \in \mathbb{C}$. The poles are called the scattering resonances which share the same multiplicity at each pole as resolvent resonances. It is a subject of great interest in mathematical physics to describe the scattering resonances approximately inside a disc of radius $r$ or in certain region in complex plane $\mathbb{C}$. Therefore, we count the poles of the meromorphically defined scattering determinant $\operatorname{det} S(\lambda)$.

In any case, we consider the determinant

$$
s(\lambda):=\operatorname{det} S(\lambda)
$$

satisfying the following properties [1-4]:

(P.1) $s(-\lambda)=\overline{s(\bar{\lambda})}=1 / s(\lambda)$;

(P.2) the point set $\mathscr{R}$ and $|s(\lambda)|$ is symmetric about the imaginary axis;

(P.3) there is no pole on the real axis except possibly a double pole at $\lambda=0$;

(P.4) there are only exceptionally finitely many poles $\left\{\mu_{1}, \ldots, \mu_{e}\right\}$ in $\mathbb{C}^{+}:=\{\mathfrak{I} z>0\}$; infinitely many poles in $\mathbb{C}^{-}:=\{\Im z<0\}$;

(P.5) the functional determinant $s(\lambda)$ is of order 3 , the number of space dimension. 
The growth estimate on $s(\lambda)$ has only upper bound as proved in [2] which is an optimal upper bound. The actual lower bound is unknown to the author. (P.5) is the most nontrivial hypothesis.

Let us define

$$
\mathfrak{S}(\lambda):=z^{m_{0}} \prod_{j=1}^{e} \frac{\lambda-\mu_{j}}{\lambda-\bar{\mu}_{j}} s(\lambda),
$$

where $0 \leq m_{0} \leq 2$ is chosen minimally such that $\mathfrak{g}(\lambda)$ has no zero at $z=0$. Surely, $\mathfrak{g}(\lambda)$ is a regular function of order three in $\overline{\mathbb{C}}^{+}:=\{\Im z \geq 0\}$. Because of (P.1), the zeros of $\mathfrak{g}(\lambda)$ are substitutes in the study of poles of $s(\lambda)$ in $\mathbb{C}^{-}:=\{\mathfrak{I} z<0\}$.

As suggested by (P.4), there are infinitely many zeros of $\mathfrak{S}(\lambda)$ in $\mathbb{C}^{+}$. We will use Cartwright's theory [5-10] to describe the zeros of $\mathfrak{g}(\lambda)$ more precisely. We state the following result.

Theorem 1. Let $n(r, \alpha, \beta, \mathfrak{g})$ be the number of the zeros of $\mathfrak{s}(\lambda)$ inside the sector $\alpha \leq \arg \lambda \leq \beta$ and $|\lambda| \leq r$. Let $h(\theta)$ be the generalized indicator function of $\mathfrak{g}(\lambda)$ with respect to proximate order $\rho(r)$. We assume the properties (P.1) to (P.5). Then, $\mathfrak{S}(\lambda)$ is of completely regular growth in $[0, \pi]$ and the following asymptotics hold:

$$
\begin{array}{r}
n\left(r, 0, \frac{\pi}{3}-\epsilon, \mathfrak{g}\right) \sim 0 r^{3(r)}+\cdots, \quad \text { as } r \longrightarrow \infty ; \\
n\left(r, \frac{\pi}{3}-\epsilon, \frac{\pi}{3}+\epsilon, \mathfrak{I}\right) \sim \frac{1}{2 \pi}\left[h\left(\frac{\pi}{6}\right)+h\left(\frac{\pi}{2}\right)\right] r^{3(r)}+\cdots, \\
\text { as } r \longrightarrow \infty ;
\end{array}
$$

$$
\begin{array}{r}
n\left(r, \frac{\pi}{3}+\epsilon, \frac{2 \pi}{3}-\epsilon, \mathfrak{g}\right) \sim 0 r^{3(r)}+\cdots, \quad \text { as } r \longrightarrow \infty \\
n\left(r, \frac{2 \pi}{3}-\epsilon, \frac{2 \pi}{3}+\epsilon, \mathfrak{g}\right) \sim \frac{1}{2 \pi}\left[h\left(\frac{\pi}{2}\right)+h\left(\frac{5 \pi}{6}\right)\right] r^{3(r)}+\cdots, \\
\text { as } r \longrightarrow \infty ;
\end{array}
$$

$$
n\left(r, \frac{2 \pi}{3}+\epsilon, \pi, \mathfrak{g}\right) \sim 0 r^{3(r)}+\cdots, \quad \text { as } r \longrightarrow \infty .
$$

The definition of a proximate order $\rho(r)$ of a regular function is to be given in Definition 3 and we define the generalized indicator function $h(\theta)$ in Definition 4. The connection of Cartwright's theory to the location of resonances is firstly mentioned in [11] and followed by $[12,13]$. In [11, page 278], Zworski studied the resonances using the theory of zeros of certain Fourier transform developed by Cartwright and Titchmarsh. In [13, page 269], Froese computed the indicator function in one dimensional potential scattering and used the fundamental theorem on the distribution of the zeros of a function of completely regular growth $[9$, page $152]$ to prove his results. In [12], this fundamental theorem is applied to study the location of resonances in sectors. In this paper, we study the indicator function and then the density function for the scattering resonances.
The existence of infinitely many resonances is known in several settings. See $[1,3,4,12]$. In particular, we previously have lower bound [4] that

$$
\varlimsup_{r \rightarrow \infty} \frac{n(r, 0,2 \pi, s)}{r}>0 .
$$

\section{Cartwright's Theory}

We collect many classic theorems from $[5-10,14]$ in this section. Readers who are familiar with the references may skip it.

Definition 2. Let $f(z)$ be a regular function in $\arg z \in[\alpha, \beta]$. Let

$$
M_{f}(r):=\max _{\alpha \leq \arg z \leq \beta}\left|f\left(r e^{i \theta}\right)\right|
$$

We say $f(z)$ is a function of finite order if there exists a positive constant $k$ such that the inequality

$$
M_{f}(r)<e^{r^{k}}
$$

is valid for all sufficiently large values of $r$. The greatest lower bound of such numbers $k$ is called the order of the function $f(z)$. By the type $\sigma_{f}$ of an entire function $f(z)$ of order $\rho$, we mean the greatest lower bound of positive number $A$ for which asymptotically we have

$$
M_{f}(r)<e^{A r^{\rho}} .
$$

That is,

$$
\sigma_{f}:=\limsup _{r \rightarrow \infty} \frac{\ln M_{f}(r)}{r^{\rho}} .
$$

If $0<\sigma_{f}<\infty$, then we say $f(z)$ is of normal type or mean type.

Definition 3. Let $\rho \in \mathbb{R}$ and $\rho(r): \mathbb{R}^{+} \rightarrow \mathbb{R}^{+}$. We say $\rho(r)$ is a Lindelöf proximate order to $\rho$ if

$\rho(r)$ is real, continuous, and piecewise differentiable

for $r>l>0$;

$$
\begin{gathered}
\lim _{r \rightarrow \infty} \rho(r)=\rho \geq 0 ; \\
\varlimsup_{r \rightarrow \infty} \frac{\log M_{f}(r)}{r^{\rho(r)}}=1 ; \\
\lim _{r \rightarrow \infty} r \rho^{\prime}(r) \log r=0,
\end{gathered}
$$

where $\rho^{\prime}(r)$ is a right- or left-hand derivative wherever different.

This is found in [8, page 54]. The following can be found in [6, page 437] and [10, page 53]. 
Definition 4. Let $f(z)$ be a regular function of proximate order $\rho(r)$ in the angle $\left[\theta_{1}, \theta_{2}\right]$. The following quantity is called the generalized indicator of the function $f(z)$ :

$$
h_{f}(\theta):=\limsup _{r \rightarrow \infty} \frac{\ln \left|f\left(r e^{i \theta}\right)\right|}{r^{\rho(r)}}, \quad \theta_{1} \leq \theta \leq \theta_{2} .
$$

We say $h_{f}(\theta)$ is sinusoidal at $\theta=\alpha$ if

$$
h_{f}=C \cos \rho(\theta-\gamma),
$$

for some constant $C$ and for $\alpha-\delta \leq \theta \leq \alpha+\delta$, where $\delta>0$; moreover, we say a function $H(\theta)$ is sinusoidal or $\rho$ trigonometric if

$$
H(\theta)=A \cos \rho \theta+B \sin \rho \theta, \quad A, B \text { are constants. }
$$

Definition 5. A function $K(\theta)$ is called $\rho$-trigonometrically convex on the closed interval $[\alpha, \beta]$ if for $\alpha \leq \theta_{1}<\theta_{2} \leq \beta$, $0<\theta_{2}-\theta_{1}<\pi / \rho$, the identities

$$
K\left(\theta_{1}\right)=h_{1}, \quad K\left(\theta_{2}\right)=h_{2}
$$

imply the inequality

$$
K(\theta) \leq H(\theta), \quad \theta_{1} \leq \theta \leq \theta_{2},
$$

where $H(\theta)$ is a $\rho$-trigonometric function such that $H\left(\theta_{1}\right)=$ $h_{1}, H\left(\theta_{2}\right)=h_{2}$. For such a pair of $h_{1}, h_{2}, H(\theta)$ is uniquely expressed by the formula

$$
H(\theta)=\frac{h_{1} \sin \rho\left(\theta_{2}-\theta\right)+h_{2} \sin \rho\left(\theta-\theta_{1}\right)}{\sin \rho\left(\theta_{2}-\theta_{1}\right)}, \quad \theta_{1} \leq \theta \leq \theta_{2} .
$$

In particular, we have

$$
h_{f}(\theta)+h_{f}\left(\theta+\frac{\pi}{\rho}\right) \geq 0
$$

wherever it makes sense. We must emphasize that a sinusoidal function $H(\theta)$ assuming $h_{1}, h_{2}$ at $0<\theta_{2}-\theta_{1}<\pi / \rho$ is uniquely determined. See [10, pages 53-54].

Theorem 6. Let $f(z)$ be a holomorphic function and satisfy (16) inside an angle. Then, its indicator function $h_{f}(\theta)$ with respect order $\rho$ is a continuous and $\rho$-trigonometrically convex function inside the angle.

Definition 7. Let $f(z)$ be an integral function of proximate order $\rho(r)$. We use

$$
n(r, \alpha, \beta, f)
$$

to denote the number of the zeros of $f(z)$ inside the sector with angle $[\alpha, \beta]$ and $0<l<|z| \leq r$; we define the density function of index $\rho(r)$ :

$$
\Delta_{f}(\alpha, \beta):=\limsup _{r \rightarrow \infty} \frac{n(r, \alpha, \beta, f)}{r^{\rho(r)}} .
$$

Definition 8. An entire function $f(z)$ of proximate order $\rho(r)$ is said to be of completely regular growth if there is some zero relative measure set $E_{0}$ such that when $r \rightarrow \infty$ outside $E_{0}$, the function $h_{f, r}(\theta)=\ln \left|f\left(r e^{i \theta}\right)\right| / r^{\rho(r)}$ tends to $h_{f}(\theta)$ uniformly.

We review Cartwright's theory for entire functions of finite order.

Definition 9. Let $f(z)$ be a regular function of proximate order $\rho(r)$ of type $\sigma_{f}$ in angle $[\alpha, \beta]$. We say $\arg z=\gamma$ is a direction of Borel of approximate order $\rho(r)$ of $f(z)$ if

$$
\begin{array}{r}
\lim _{r \rightarrow \infty} \frac{n(r, \gamma-\epsilon, \gamma+\epsilon, f-a)}{r^{\rho(r)}}=\eta(\epsilon)>0, \\
\forall \epsilon>0, \alpha<\gamma<\beta,
\end{array}
$$

and for all values $a$, except perhaps one.

If $0<\sigma_{f}<\infty$, then we say $\arg z=\gamma$ is a direction of Borel of maximum kind.

We review the following Cartwright theorem [5, page 504, Theorem A; page 507, Theorem V] or, more generally, as in [9, page 155]. However, one should notice the typography in the corollary on page 155 . The density of the zero set inside an open angle with a sinusoidal indicator function is zero. We will examine the condition for a direction of Borel has an exceptional value or not.

Theorem 10. Suppose that $f(z)$ is an integral function of proximate order $\rho(r)$, where $\rho>1$, and that

$$
h(\theta)=h(0) \cos \theta \rho, \quad|\theta| \leq \frac{\pi}{2 \rho} .
$$

Then, for any $\delta>0$,

$$
\lim _{r \rightarrow \infty} \frac{n(r,-\pi / 2 \rho+\delta, \pi / 2 \rho-\delta, f)}{r^{\rho(r)}}=0 .
$$

In particular, $f(z)$ has no direction of Borel of proximate order $\rho(r)$ inside $|\theta|<\pi / 2 \rho$.

There is another theorem for the nonexistence of direction of Borel. See [14, page 201].

Theorem 11. Let $\gamma \in(\alpha, \beta)$ and $h(\theta) \leq 0$ for all $\theta \in(\alpha, \beta)$, then $\gamma$ is not a direction of Borel for which with respect to $\rho(r)$.

Theorem 12. If $f(z)$ is of finite proximate order $\rho(r)$ for $\alpha \leq$ $\arg z \leq \beta,|z| \geq l$, where $\beta-\alpha>\pi / \rho$, then there is at least one direction of Borel of maximum kind for which $\alpha<\arg z<\beta$.

This is stated the same as in [6, page 425]. We state the following fundamental theorem in [9, page 152].

Theorem 13. If a holomorphic function $F(z)$ of order $\rho(r)$ has completely regular growth within an angle $\left(\theta_{1}, \theta_{2}\right)$, then for all 
values $9, \theta$ such that $\theta_{1}<\vartheta<\theta<\theta_{2}$, except possibly for a denumerable set, the following limit exists:

$$
\frac{1}{2 \pi \rho} s_{F}(\vartheta, \theta)=\lim _{r \rightarrow \infty} \frac{n(r, \vartheta, \theta, F)}{r^{\rho(r)}},
$$

where

$$
s_{F}(\vartheta, \theta)=h_{F}^{\prime}(\theta)-h_{F}^{\prime}(\vartheta)+\rho^{2} \int_{\vartheta}^{\theta} h_{F}(\varphi) d \varphi .
$$

The exceptional denumerable set can only consist of points for which $h_{F}^{\prime}(\theta+0) \neq h_{F}^{\prime}(\theta-0)$.

We may find $h(\theta)$ by the following lemma in [9, page 464].

Lemma 14. Let $f(z)$ be a holomorphic function of proximate order $\rho(r)$ inside the angle $|\arg z| \leq \alpha, 0<\alpha \leq \pi / 2 \rho$ and let $h( \pm \alpha)=h(0) \cos \rho \alpha$. Then, $h(\theta)=h(0) \cos \rho \theta,-\alpha \leq \theta \leq \alpha$.

\section{Proof of Theorem 1}

Using assumption (P.5), we have $\rho=3$. We use Theorem 6 to have that

$$
\begin{gathered}
h(\theta) \leq C \cos \rho\left(\theta-\frac{\pi}{2}\right)=: H(\theta), \quad \text { for some } C \\
h\left(\theta_{j}\right)=H\left(\theta_{j}\right), \quad j=1,2,
\end{gathered}
$$

where $\pi / 2-\pi / 2 \rho \leq \theta_{1}<\theta_{2} \leq \pi / 2+\pi / 2 \rho$ and $0<\theta_{2}-\theta_{1}<$ $\pi / \rho$. Because of the continuity of $h(\theta)$,

$$
h(\theta) \leq C \cos 3\left(\theta-\frac{\pi}{2}\right), \quad \theta \in\left[\frac{\pi}{3}, \frac{2 \pi}{3}\right] .
$$

By the symmetry of $|\mathfrak{s}(\lambda)|$ along the imaginary axis, its indicator function $h(\theta)$ has a local minimum or maximum at $\theta=\pi / 2$. We use the $\rho$-trigonometric convexity of $h(\theta)$ in [9, page 56] which implies that

$$
h(\theta) \geq h\left(\frac{\pi}{2}\right) \cos 3\left(\theta-\frac{\pi}{2}\right), \quad \forall \theta \in\left[\frac{\pi}{3}, \frac{2 \pi}{3}\right] .
$$

Therefore, (34) and (35) imply that

$$
h\left(\frac{\pi}{3}\right)=h\left(\frac{2 \pi}{3}\right)=0
$$

We apply the Lemma 14 to obtain

$$
h(\theta)=h\left(\frac{\pi}{2}\right) \cos 3\left(\theta-\frac{\pi}{2}\right), \quad \forall \theta \in\left[\frac{\pi}{3}, \frac{2 \pi}{3}\right] .
$$

Once again we can apply Lemma 14 to the intervals $[0, \pi / 3]$ and $[2 \pi / 3, \pi]$. The equalities

$$
h(0)=h\left(\frac{\pi}{3}\right)=h\left(\frac{2 \pi}{3}\right)=h(\pi)=0
$$

imply that

$$
h(\theta)= \begin{cases}-h\left(\frac{\pi}{6}\right) \cos 3\left(\theta-\frac{\pi}{2}\right), & \text { for } \theta \in\left[0, \frac{\pi}{3}\right] \\ h\left(\frac{\pi}{2}\right) \cos 3\left(\theta-\frac{\pi}{2}\right), & \text { for } \theta \in\left[\frac{\pi}{3}, \frac{2 \pi}{3}\right] \\ -h\left(\frac{\pi}{6}\right) \cos 3\left(\theta-\frac{\pi}{2}\right), & \text { for } \theta \in\left[\frac{2 \pi}{3}, \pi\right] .\end{cases}
$$

Therefore, $\mathfrak{s}(\lambda)$ is of completely regular growth in $(0, \pi / 3)$, $(\pi / 3,2 \pi / 3)$, and $(2 \pi / 3, \pi)$ and, hence, in $(0, \pi)$ by applying directly from Definition 8 . In general, from $\rho$-trigonometric convexity (25), one has

$$
h\left(\frac{\pi}{2}\right)+h\left(\frac{\pi}{6}\right) \geq 0 .
$$

We apply Theorem 10 with indicator $(39)$ in $(0, \pi / 3),(\pi / 3$, $2 \pi / 3)$, and $(2 \pi / 3, \pi)$, respectively. The set of zeros of $\mathfrak{g}(\lambda)$ is of density zero there. This proves the asymptotics (8), (10), and (12).

Let us discuss the following four possible types of conjunction in (39):

$$
\begin{array}{ll}
h\left(\frac{\pi}{2}\right)>0, & h\left(\frac{\pi}{6}\right)>0 ; \\
h\left(\frac{\pi}{2}\right)>0, & h\left(\frac{\pi}{6}\right) \leq 0 ; \\
h\left(\frac{\pi}{2}\right) \leq 0, & h\left(\frac{\pi}{6}\right)>0 ; \\
h\left(\frac{\pi}{2}\right) \leq 0, & h\left(\frac{\pi}{6}\right) \leq 0 .
\end{array}
$$

For the case (44), we conclude that $h(\theta) \leq 0$, for $\theta \in[0, \pi]$, by (39). However, Theorems 12 and 11 exclude this case.

For the case (41), (42), and (43), we apply Theorem 10, which suggests that $0, \pi / 3,2 \pi / 3, \pi$ are the only four possible directions of Borel. Now we apply the formula (32):

$$
\begin{aligned}
s\left(\frac{\pi}{6}, \frac{\pi}{2}\right) & =h^{\prime}\left(\frac{\pi}{2}\right)-h^{\prime}\left(\frac{\pi}{6}\right)+9 \int_{\pi / 6}^{\pi / 2} h(\varphi) d \varphi \\
& =9 \int_{\pi / 6}^{\pi / 2} h(\varphi) d \varphi \\
& =3\left[h\left(\frac{\pi}{6}\right)+h\left(\frac{\pi}{2}\right)\right] ; s\left(\frac{\pi}{2}, \frac{5 \pi}{6}\right) \\
& =3\left[h\left(\frac{\pi}{2}\right)+h\left(\frac{5 \pi}{6}\right)\right] .
\end{aligned}
$$

Therefore,

$$
\begin{aligned}
\lim _{r \rightarrow \infty} \frac{n(r, \pi / 6, \pi / 2, \mathfrak{g})}{r^{3(r)}} & =\frac{1}{2 \pi}\left[h\left(\frac{\pi}{6}\right)+h\left(\frac{\pi}{2}\right)\right] ; \\
\lim _{r \rightarrow \infty} \frac{n(r, \pi / 2,5 \pi / 6, \mathfrak{I})}{r^{3(r)}} & =\frac{1}{2 \pi}\left[h\left(\frac{\pi}{2}\right)+h\left(\frac{5 \pi}{6}\right)\right] .
\end{aligned}
$$

This proves (9) and (11). 


\section{Conflict of Interests}

The author declares that there is no conflict of interests regarding the publication of this paper.

\section{References}

[1] J. Sjöstrand and M. Zworski, "Lower bounds on the number of scattering poles. II," Journal of Functional Analysis, vol. 123, no. 2, pp. 336-367, 1994.

[2] G. Vodev, "Sharp bounds on the number of scattering poles for perturbations of the Laplacian," Communications in Mathematical Physics, vol. 146, no. 1, pp. 205-216, 1992.

[3] M. Zworski, "Poisson formulae for resonances," in Séminaire sur les Équations aux Dérivées Partielles, 1996-1997, École Polytechnique, Palaiseau, France, 1997.

[4] A. Sá Barreto, "Remarks on the distribution of resonances in odd dimensional Euclidean scattering," Asymptotic Analysis, vol. 27, no. 2, pp. 161-170, 2001.

[5] M. L. Cartwright, "On the directions of Borel of functions which are regular and of finite order in an angle," Proceedings of the London Mathematical Society 2, vol. 38, no. 1, pp. 503-541, 1933.

[6] M. L. Cartwright, "On the directions of Borel of analytic functions," Proceedings of the London Mathematical Society 2, vol. 38, no. 1, pp. 417-457, 1933.

[7] M. L. Cartwright, "On functions which are regular and of finite order in an angle," Proceedings of the London Mathematical Society 2, vol. 38, no. 1, pp. 158-179, 1933.

[8] M. L. Cartwright, Integral Functions, Cambridge University Press, Cambridge, UK, 1956.

[9] B. Ja. Levin, Distribution of Zeros of Entire Functions, American Mathematical Society, Providence, RI, USA, 1972.

[10] B. Ya. Levin, Lectures on Entire Functions, vol. 150, American Mathematical Society, Providence, RI, USA, 1996.

[11] M. Zworski, "Distribution of poles for scattering on the real line," Journal of Functional Analysis, vol. 73, no. 2, pp. 277-296, 1987.

[12] T. J. Christiansen, "Schrödinger operators and the distribution of resonances in sectors," Analysis \& PDE, vol. 5, no. 5, pp. 961982, 2012.

[13] R. Froese, "Asymptotic distribution of resonances in one dimension," Journal of Differential Equations, vol. 137, no. 2, pp. 251272, 1997.

[14] D. Drasin and A. Weitsman, "On the Julia directions and Borel directions of entire functions," Proceedings of the London Mathematical Society, vol. 32, no. 2, pp. 199-212, 1976. 


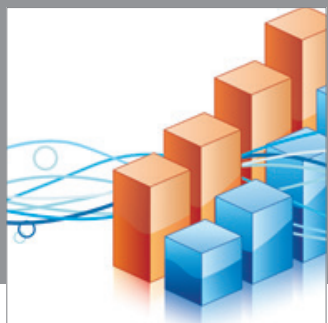

Advances in

Operations Research

mansans

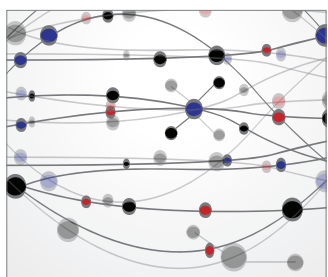

The Scientific World Journal
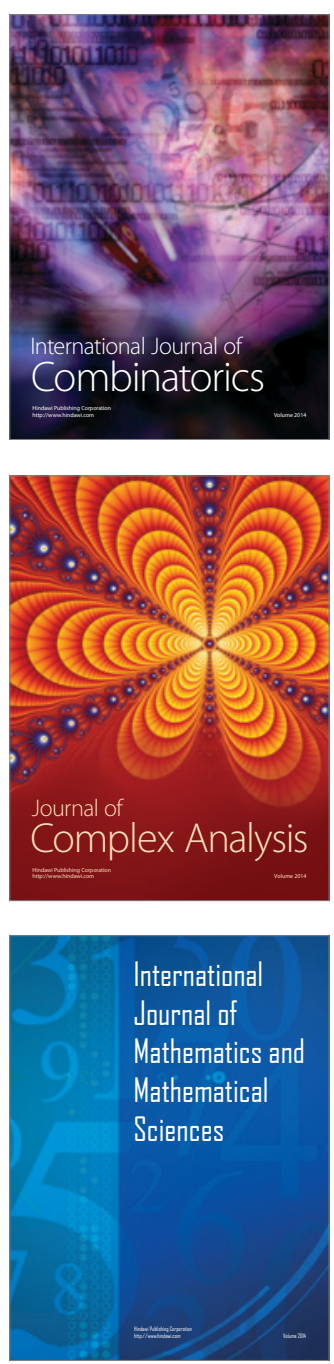
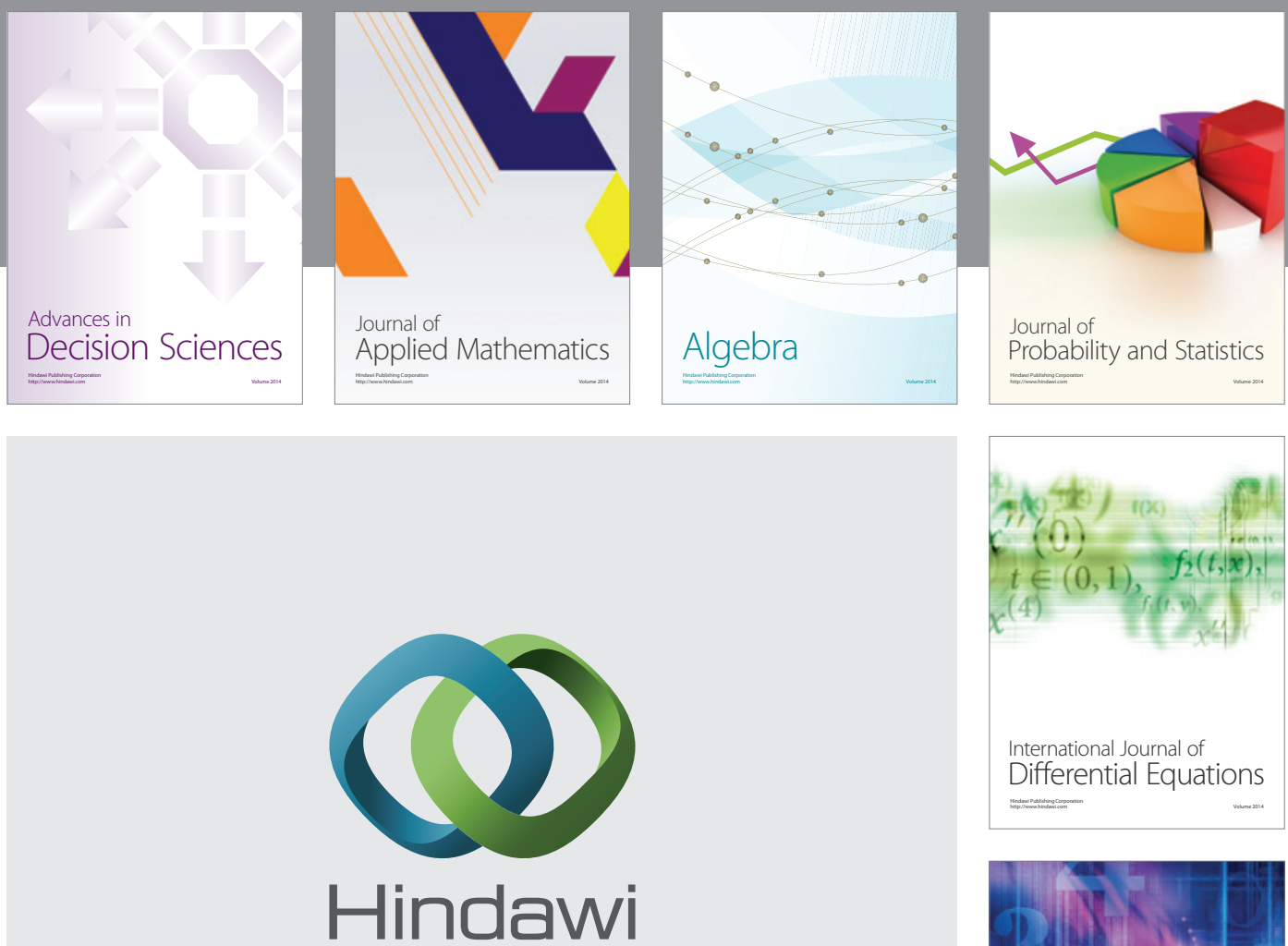

Submit your manuscripts at http://www.hindawi.com
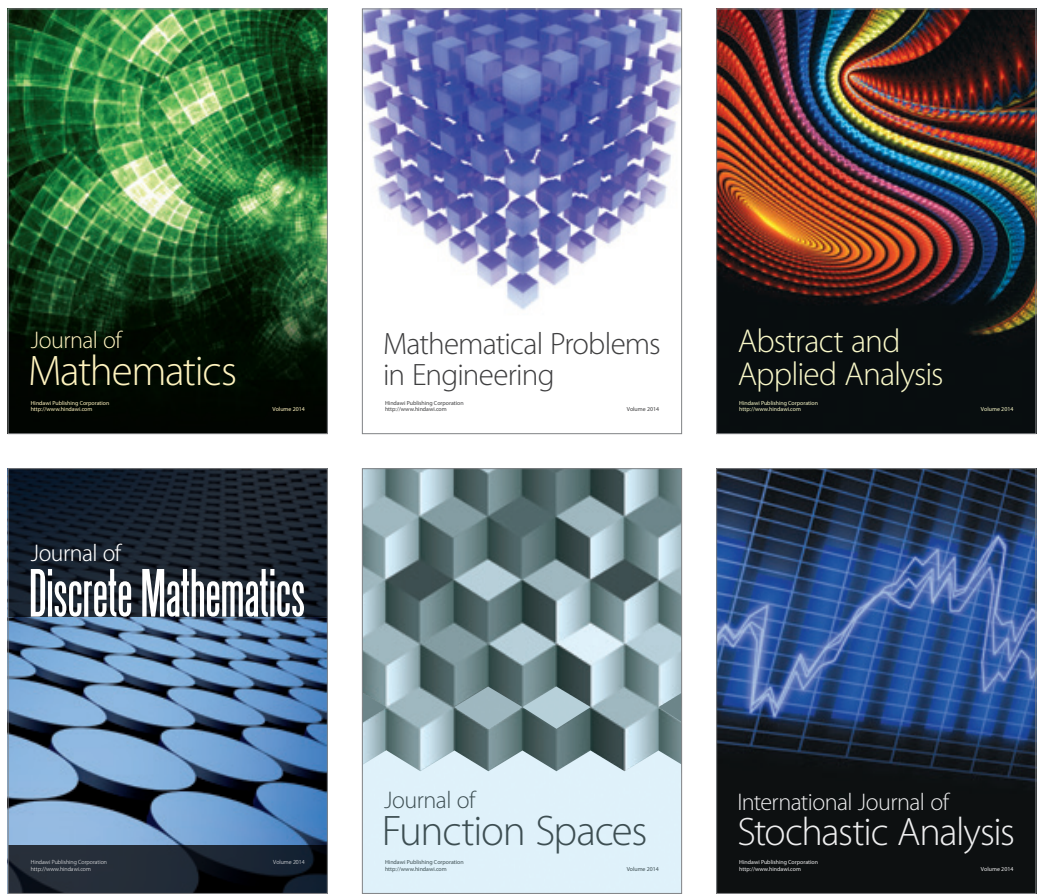

Journal of

Function Spaces

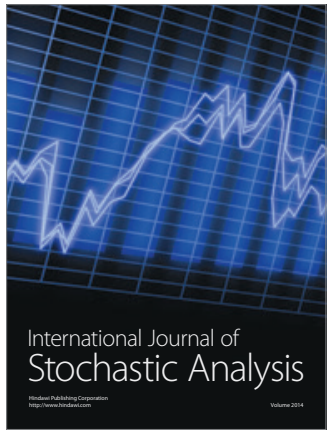

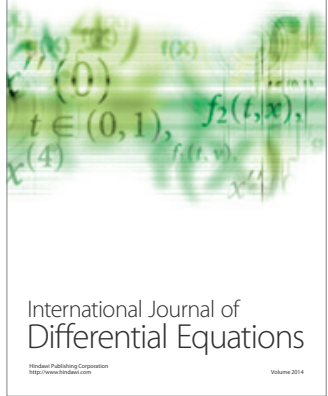
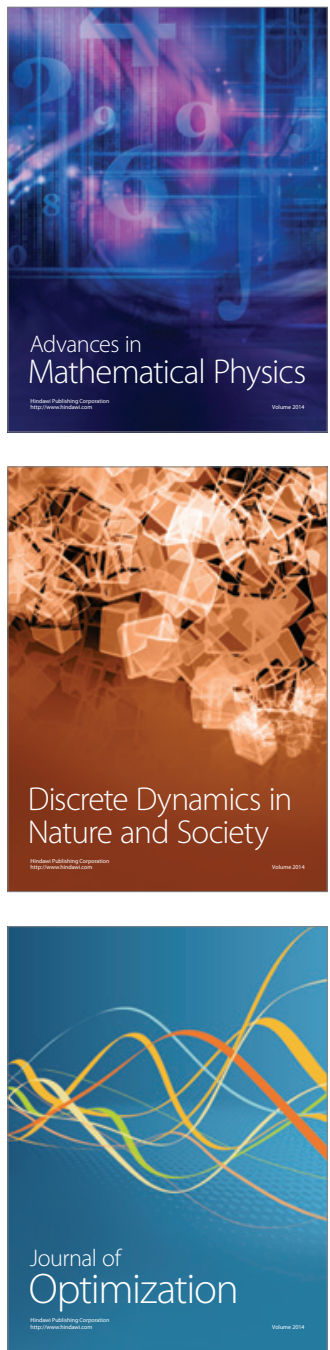